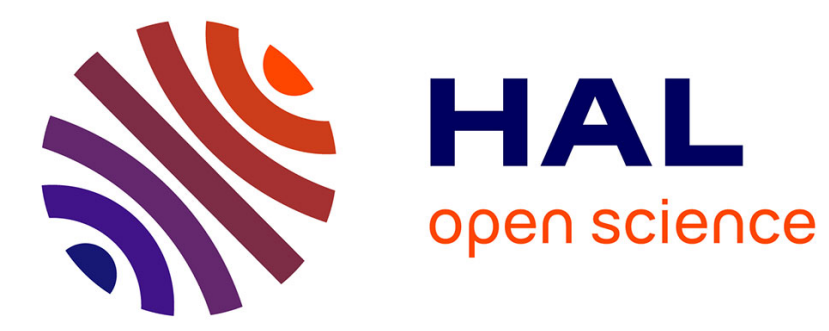

\title{
L'isotropie de la loi de Hubble testée sur le champ des Quasars. Corrélation avec le superamas local H. Reboul
}

\section{To cite this version:}

H. Reboul. L'isotropie de la loi de Hubble testée sur le champ des Quasars. Corrélation avec le superamas local. Annales de Physique, 1979, 4, pp.213-215. 10.1051/anphys/197904040213 . hal02160306

\section{HAL Id: hal-02160306 https://hal.science/hal-02160306}

Submitted on 19 Jun 2019

HAL is a multi-disciplinary open access archive for the deposit and dissemination of scientific research documents, whether they are published or not. The documents may come from teaching and research institutions in France or abroad, or from public or private research centers.
L'archive ouverte pluridisciplinaire HAL, est destinée au dépôt et à la diffusion de documents scientifiques de niveau recherche, publiés ou non, émanant des établissements d'enseignement et de recherche français ou étrangers, des laboratoires publics ou privés. 


\section{L'isotropie de la loi de Hubble testée sur le champ des Quasars. Corrélation avec le superamas local}

\section{H. Reboul}

Laboratoire d'Astronomie. Université des Sciences et Techniques du Languedoc. Place Eugène Batailion F34060 Montpellier Cédex

Summary. A test of the isotropy of Hubble law on the field of quasars - Correlation with the local supercluster. Selecting quasars on the ground of intrinsic parameters makes a first test of the isotropy of Hubble law possible. Hemispheric anisotropy of $H_{0}$ is not significant and has to be less than $-25 \%,+34 \%$. A correlation with the geometry of the Local Supercluster is discussed.

Résumé. La sélection des quasars sur des critères intrinsèques permet une première approche du degré d'isotropie de la loi de Hubble. L'anisotropie hémisphérique éventuelle est majorée à $-25 \%$ et $+34 \%$. Une corrélation avec la géométrie du superamas local est discutée.

Le module de Hubble classique, $H M$, est défini (Rubin et al. 1973) par :

$$
H M=\log c z-0,2 \mathrm{~m}=\log H_{0}-0,2 M-5
$$

Dans le cadre d'un modèle de Friedmann, il est possible de déduire un module de Hubble généralisé, $H M^{*}$, sous la forme :

$$
H M^{*}=\log \left\{c \cdot\left(q_{0}\right)^{-2} \cdot\left(q_{0} z+\left(q_{0}-1\right)\left[\left(1+2 q_{0} z\right)^{1 / 2}-1\right]\right)\right\}-0,2 \mathrm{~m}
$$

On peut vérifier aisément que :

$$
H M^{*}=\log H_{0}-0,2 M-5 .
$$

Ce module, $H M^{*}$, a été utilisé pour tester l'isotropie de la relation de Hubble - ou plus exactement celle de Mattig - sur le champ des quasars.

En accord avec les travaux de Setti et Woltjer (1973 a,b) et de Stannard (1973), 132 objets du catalogue de Smith-Haeni (1977) ont été sélectionnés sur les critères d'indices spectraux radio entre 408 et $1415 \mathrm{MHz}:-\alpha \geqslant 0,7$ ou $|\alpha| \leqslant 0,3$.

On a appliqué aux magnitudes les corrections de couleur, les corrections d'absorption galactique (De Vaucouleurs, 1976) et les K-corrections (Evans et Hart, 1977).

Pour les 132 quasars, les $H M^{*}$ ont été calculés en fonction de la magnitude corrigée, du décalage d'émission et de différentes valeurs de $q_{0}$. Pour $q_{0}=0,5$ on obtient une moyenne : $\left\langle H M^{*}\right\rangle_{132}=1,91$ et un écart-type $\sigma=0,255$, ce qui correspond à $\langle M\rangle_{132}=-25,19 \pm 1,27$, si $H=75 \mathrm{~km} \cdot \mathrm{s}^{-1} \cdot \mathrm{Mpc}^{-1}$.

La figure 1 montre la répartition spatiale des 132 quasars en coordonnées supergalactiques. Une recherche systématique d'anisotropie a été ensuite effectuée en coupant la sphère céleste en deux hémisphères I et II et en calculant la différence

$$
\left.\Delta H M^{*}=\left\langle H M^{*}\right\rangle_{\mathrm{I}}-<H M^{*}\right\rangle_{\mathrm{II}} .
$$

Le plan de la partition a été varié de $10^{\circ}$ en $10^{\circ}$ selon les deux dimensions angulaires. 


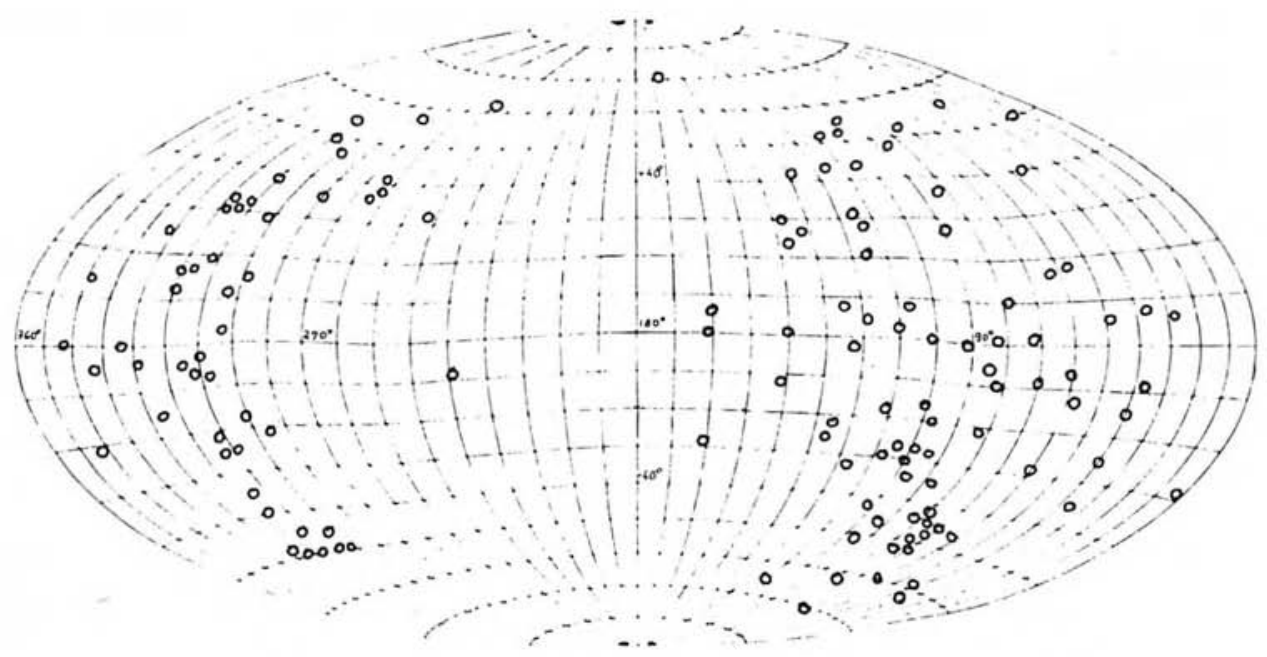

FIG. 1. - Répartition des 132 quasars $(-\alpha \leqslant 0,7$ ou $|\alpha| \leqslant 0,3)$ en coordonnées supergalactiques.

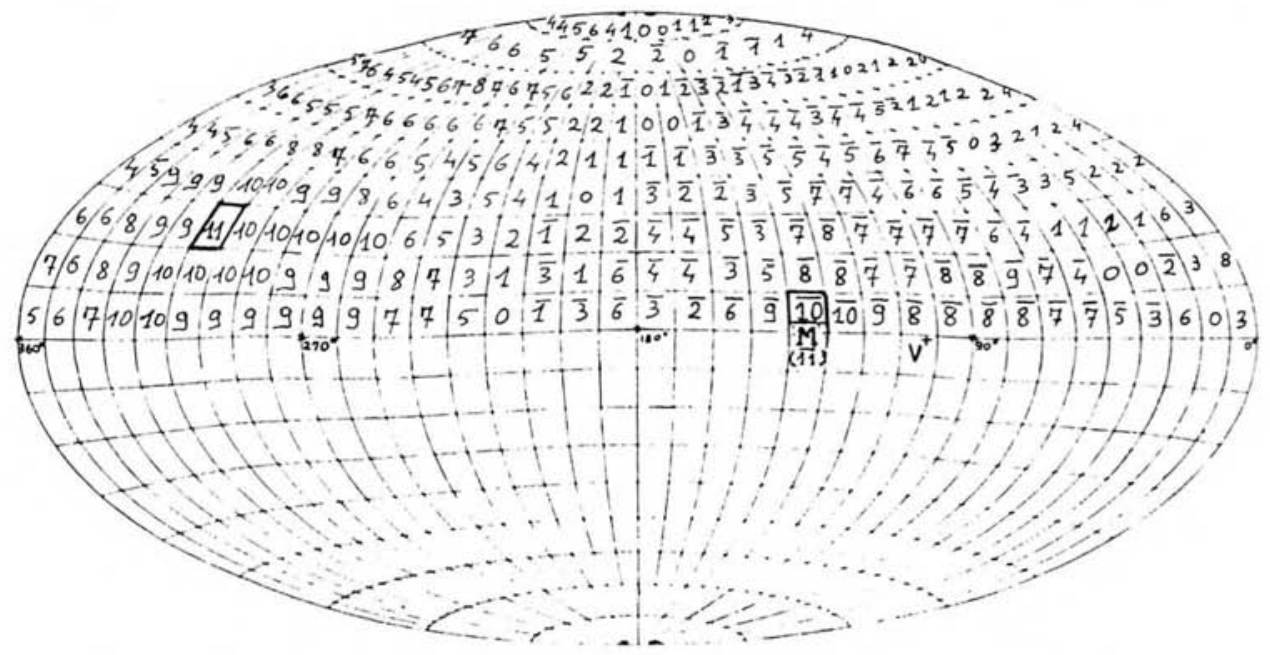

FIG. 2. - Répartition de l'anisotropie hémisphèrique $\left(\left\langle H M^{*}\right\rangle_{I}-\left\langle H M^{*}\right\rangle_{I I}\right)$ en coordonnées supergalactiques pour le modele de Friedmann $\left(q_{0}=0,5\right)$.

La figure 2 montre, en coordonnées supergalactiques, le résultat de cette investigation pour $q_{0}=0,5$. Le chiffre dans chaque case, multiplié par $10^{-2}$ donne le $\Delta H M^{*}$ lorsque le pôle de l'hémisphère I est au centre de la case.

Les autres valeurs de $q_{0}$ ont conduit à des résultats semblables en direction et en grandeur d'anisotropie.

Pour tester la signification des extrema d'anisotropie observés, on a utilisé des processus de simulation en gardant les coordonnées des objets réels et en remplaçant leurs $H M^{*}$ par des nombres aléatoires gaussiens de même écart-type.

Les deux résultats essentiels de ces simulations sont :

- l'anisotropie observée à 6 chances sur 10 d'être due au hasard. 
- à un taux de confiance 0,95 , on peut majorer l'éventuelle anisotropie hémisphérique de $H_{0}$ par :

$$
H_{0}\left\{\begin{array}{l}
+34 \% \\
-25 \%
\end{array}\right.
$$

Ceci est le résultat d'une étude a priori. A posteriori, on remarque la faible latitude supergalactique du minimum de $H M^{*}$.

Une étude ultérieure, avec le pôle de la partition dans le plan du superamas, a permis d'obtenir un extremum encore plus élevé que celui dégagé par la première étude. On enregistre ainsi $\Delta H M^{*}=-0,1061$ lorsque le pôle de l'hémisphère I est à $\mathrm{SGB}=0^{\circ}$ et $\mathrm{SGL}=135^{\circ}$ (point M sur la figure 2).

On ne peut que noter la corrélation de position entre le minimum de $H M^{*}$ et le centre du superamas, l'amas Virgo $\left(\mathrm{SGB}=-2^{\circ}, \mathrm{SGL}=104^{\circ}\right)$, représenté par le point $\mathrm{V}$ sur la figure 2.

Y a-t-il une absorption de $\sim 0,5$ magnitude à travers le centre et le disque du superamas ? Un test de Student donnerait un taux de confiance supérieur à 0,9 à cette interprétation mais son emploi est ici totalement illégitime étant donné le mélange d'a priori et d'a posteriori de la démarche.

Seule l'étude d'autres échantillons permettrait de résoudre l'alternative : fluctuation, statistique, absorption supergalactique, sous l'hypothèse d'isotropie de $H_{0}$.

Note : Un compte-rendu plus détaillé de la présente étude et de ses résultats est actuellement en préparation.

\section{Bibliographie}

De Vaucouleurs (G.), De Vaucouleurs (A.), Corwin (H.G.Jr). - Second Reference Catalogue of Bright Galaxies". University of Texas Press.,1976.

Evans (A.), Hart (D.) - Astron. Astrophys., 1977, 58, 241.

Rubin (V.C.), Ford (W.K. Jr.), Rubin (J.S.). - Astrophys J., 1973, 183, L 111.

Setti (G.), Woltjer (L.) - Ann. New. York Acad. Sci., 1973 a, 224, 8

Setti (G.), Woltjer (L.) - Astrophys J., 1973, 181, L 61.

Smith-Haeni (A.L.) - Astron. Astrophys. (Suppl. Ser), 1977, 27, 205.

Stannard (D.) - Nature, 1973, 246, 295. 